\title{
Prioritized neural computations of socially-relevant signals during perceptual decision-making
}

Abbreviated title (50-character maximum): Prioritized neural computations of social signals

Meaux E. ${ }^{1}$, El Zein M. ${ }^{2}$, Mennella R. ${ }^{1}$, Wyart V. $^{1^{*}}$ \& Grèzes J. ${ }^{1^{*}}$

* shared senior authorship

1. Cognitive and Computational Neurosciences Laboratory (LNC ${ }^{2}$ ), INSERM U960, DEC, Ecole Normale Supérieure, PSL University, 75005, Paris, France

2. Institute of Cognitive Neuroscience, University College London, 17 Queen Square, London, WC1N $3 A R$

Corresponding author: Emilie Meaux (meauxemilie@gmail.com) \& Julie Grèzes (julie.grezes@ens.fr)

- Number of pages: 32

- Number of figures: 5 (and 5 supplementary figures)

- Number of words for abstract (199), introduction (736), and discussion (1499)

- Conflict of interest statement: The authors declare no competing financial interests.

- Acknowledgments: This research was supported by FRM Team DEQ20160334878, INSERM, ENS, and the French National Research Agency under Grants ANR-10-LABX-0087 IEC and ANR-17-EURE0017 FrontCog. 


\section{Abstract}

2

3

4

5 developed a novel behavioral paradigm in which participants performed two different detection

6 tasks on the same, two-dimensional visual stimuli. We presented morphed facial displays of emotion

7 (from neutral to angry) on top of a morphed colored background (from grey to violet). Participants

8 reported the presence or absence of either emotion (anger) or color (violet) in the stimulus, while

9 ignoring the other task-irrelevant dimension. Importantly, we equalized detection sensitivity across dimensions using an adaptive titration procedure. Computational modeling of electroencephalographic (EEG) activity first revealed that premotor EEG activity scales with the amount of perceptual evidence earlier, around $150 \mathrm{~ms}$, when the decision concerns emotion rather than color. Second, participant choice was decoded earlier during emotion (260ms) than color decisions in band-limited EEG power in the same premotor regions. Third, these two effects varied across participants as a function of their social anxiety. Together, these findings indicate that emotion cues benefit from a prioritized neural coding in action-selective brain regions, further supporting their motivational value.

\section{Keywords: perceptual decisions, social, emotion, motor, computational modeling}




\section{Introduction}

2

3

4

5

6

7

8

9

As highly social species, most of human decisions are made in social contexts. Accurate decoding of socially-relevant information emitted by others is crucial to guiding adaptive decisions. Given their motivational value and relevance for successful interactions, social signals are granted preferential processing: human faces and bodies rapidly capture attention in natural scenes (Fletcher-Watson, Findlay, Leekam, \& Benson, 2008), individuals better detect changes in faces than in non-social objects (Ro, Russell, \& Lavie, 2001) and respond faster to social than to non-social hazards (Bishop, Biasini, \& Stavrinos, 2017). Within this object-relevance hierarchy, the processing of emotional expressions, especially when they signal threat, is also prioritized in comparison to neutral displays and non-social stimuli (Vuilleumier, 2005). Such preferences for and responses to social signals are manifest soon after birth (Shultz, Klin, \& Jones, 2018). While such behavioral evidence points toward a prioritized processing of socially-relevant signals, from initial orientation to behavioral execution, it remains unclear whether socially-relevant signals also engage specific neural computations during perceptual decision-making.

Perceptual decision-making defines the process by which ambiguous sensory signals are transformed into adaptive motor actions, e.g. (Hanks \& Summerfield, 2017). Perceptual decisions based on highly relevant social signals (threat stimuli) are associated with enhanced neural encoding in sensory, associative and motor regions, from $200 \mathrm{~ms}$ following stimulus presentation (El Zein, Wyart, \& Grèzes, 2015b). Such prioritization of highly relevant social signals in sensory and motor systems was suggested to: 1 . increase perceptual sensitivity to relevant features of the sensory environment, and 2. motivate rapid and adaptive behavioural responses. Intriguingly, perceptual decisions based on non-social, neutral stimuli (e.g., Gabor patterns, random-dot motion patterns) are also associated with the temporally overlapping activation of sensory, associative and motor regions, at similar latencies (Cisek \& Kalaska, 2010; Shushruth, Mazurek, \& Shadlen, 2018; Siegel, Buschman, \& Miller, 2015; van Ede, Chekroud, Stokes, \& Nobre, 2019). By reporting comparable neural mechanisms for social and non-social decisions, these findings cast doubt on the 
1 idea that the processing of socially-relevant signals is prioritized over the processing of non-social, 2 neutral signals.

3 Major differences in the tasks and stimuli used in previous research have precluded a direct 4 comparison between social and non-social decisions. Indeed, past studies used either only social 5 stimuli - i.e. emotional displays (El Zein et al., 2015b; Pessoa \& Padmala, 2005, 2007; Thielscher \& 6 Pessoa, 2007), or asked participants to categorize ambiguous stimuli as either social or non-social 7 (Delis, Onken, Schyns, Panzeri, \& Philiastides, 2016; Heekeren, Marrett, Bandettini, \& Ungerleider, 8 2004; Philiastides, Ratcliff, \& Sajda, 2006; Philiastides \& Sajda, 2006, 2007; Tu, Schneck, Muraskin, \& 9 Sajda, 2017). To our knowledge, only one experiment has directly assessed the neural overlap between social and non-social decisions (Sun, Yu, \& Wang, 2017). However, neither the amount of perceptual evidence nor the decision difficulty were equalized across social and non-social stimuli. Therefore, these past studies did not permit the characterization of specific neural mechanisms involved in the processing of socially-relevant compared to non-social stimuli.

To reach a comprehensive understanding of whether and how socially-relevant signals may be prioritized during decision-making, we have developed a novel electroencephalography (EEG) paradigm in which participants performed two different detection tasks on the same, twodimensional visual stimuli. We used facial expressions of anger as behaviorally relevant social stimuli. Indeed, by clearly signaling an impending verbal or physical assault (Sander, Grandjean, Kaiser, Wehrle, \& Scherer, 2007), angry expressions are thought to convey not only behavioral intentions to the observed person, but also strong action requests to the observer. We presented morphed facial displays (from neutral to angry) on top of a colored background (from grey to violet). In different blocks, participants were asked to report the presence or absence of either emotion (anger) or color (violet) in the stimulus, while ignoring the other task-irrelevant dimension (Figure 1). Importantly, to tightly match social (emotion) and non-social (color) perceptual decisions, we not only equalized the amount of sensory evidence in the stimuli across tasks using morph matching, but also equalized detection sensitivity (decision difficulty) across the two stimulus dimensions (emotion and color) 
1 using an adaptive Bayesian titration procedure. We expected the neural processing of angry displays,

2 that are behaviorally relevant for an individual's survival, to be prioritized concomitantly in sensory

3 and motor EEG correlates of decision-making (Conty, Dezecache, Hugueville, \& Grèzes, 2012; El Zein

4 et al., 2015b). To further isolate the specificity of social decisions, we also assessed how social

5 anxiety influences the neural mechanisms underlying the selective processing of socially-relevant

6 information.

\section{$7 \quad$ Materials and methods}

\section{$8 \quad$ Participants}

9 Thirty-eight healthy young adults (19 females; mean age $\pm s . d=26.6 \pm 2.5$ years) participated in an EEG experiment. All participants were right-handed, with a normal vision and had no neurological or psychiatric history. The experimental protocol was approved by INSERM and the local research ethics committee (Comité de protection des personnes lle de France III - Project CO7-28, $\mathrm{N}^{\circ}$ Eudract: 207-A01125-48) and it was carried out in accordance with the Declaration of Helsinki. The participants provided informed written consent and were compensated for their participation.

\section{Stimuli}

Stimuli consisted of 16 identities ( 8 females) adapted from the Radboud Faces Database (Langner et al., 2010) that varied in emotion (from neutral to angry expressions) and gaze direction (direct toward the participant or averted $45^{\circ}$ to the left or right). Using Adobe Photoshop CS5.1 (Adobe Systems, San Jose (A), faces were modified to remove any visible hair, resized and repositioned so that eyes, nose and mouth appeared within the same circumference. All images were converted to greyscale and cropped into an oval aperture presented at the center of the screen.

To vary the intensity of emotional expressions, faces were morphed from neutral to angry expressions using the morphing software FantaMorph (Abrosoft). Eight levels of morphs were created from neutral (level 0) to angry (level 7) expressions using a simple linear morphing 
1 faces were previously used in the El Zein et al. study (2015). In addition to the emotional morphing,

2 we created color masks, morphed from grey (level 0) to violet (level 7). We calibrated the morphing

3 between the emotional expressions and color masks by performing an intensity rating pre-test of the

4 emotional and color morphs and adjusting the morphs based on the results. Participants $(n=10)$ were

5 presented with the facial expressions or the color masks for $250 \mathrm{~ms}$ and rated their perceived

6 intensity on a continuous scale from "not at all intense" to "very intense" using a mouse device (with

7 a maximum of 3 seconds to respond). We adjusted for differences between emotion and color

8 morphs by linearizing the mean curves of judged intensities and creating corresponding morphs that

9 were thus equalized according to perceived intensities.

Experimental design

Using the Psychophysics-3 Toolbox (Brainard, 1997; Pelli, 1997), stimuli were projected on a black screen. Each trial was initiated with a white oval delimiting the faces that remained throughout the trial. The white oval appeared for approximately $500 \mathrm{~ms}$, followed by a white fixation point presented at eye level for approximately $1000 \mathrm{~ms}$ (to ensure a natural fixation to upcoming faces and avoid eye movements from the center of the oval to eye regions). Then, the morphed face on top of which we superposed a morphed color mask was displayed for $250 \mathrm{~ms}$. After face offset, participants had to perform either an emotion or a color decision task, according to a block design. Depending on the block, participants were asked to report ( $2 \mathrm{~s}$ max after face offset) the presence or absence of either emotion (anger - social decision) or color (violet - non-social decision) in the stimulus, while ignoring the other task-irrelevant dimension (See Figure 1). They provided their response by pressing one of the two buttons localized on two external devices held in their right and left hands, with their right or left index correspondingly. A grey/violet and neutral/anger button mapping was used (4 possible hand mappings), kept constant within subjects and counterbalanced across subjects. The experiment was divided into 32 experimental blocks ( 16 blocks with emotion decisions, 16 blocks with color decisions), each consisting of 32 trials, balanced in the number of gaze (2), gender (2) and morph 
11024 trials for the entire experiment. Participants alternated between emotion and color decisions at

2 each block.

\section{Procedure}

4

Upon arrival at the laboratory, the participants read and signed an informed consent form. Participants were then seated in a dimly lit, sound-attenuated room and EEG electrodes were attached. Participants were given the instructions for the emotion and color tasks, and were also instructed to maintain fixation and avoid blinking during picture presentation.

Prior to the experiment, each participant undertook short practice blocks (one emotion block and one color block, 2 IDs, 16 trials each). Importantly, to ensure that the observed differences at the neural level between tasks did not depend on differences in difficulty, we equalized detection sensitivity across emotion and color dimensions using an adaptive Bayesian titration procedure. This two block training session was followed by titration blocks (one emotion block and one color block, 2 IDs, 32 trials each) during which each participant's perceptual sensitivity to both emotion and color dimensions was estimated separately. The choices of each participant were regressed against the morph level of emotion or color in a general linear model (GLM) and parameter estimates were extracted for each dimension. These parameters estimates were then used to calculate a titration factor (i.e. multiplier) by which the morph of the color mask applied on the face was multiplied to rescale the color level of the stimulus to the same perceptual intensity as the emotion level. This procedure was reiterated every two blocks across the experiment. This titration procedure ensured that the difficulty between emotion and color decisions was calibrated and updated across the experiment, such that the same psychometric function slope reflected participants' behavior in both conditions.

To maintain motivation, participants were informed prior to the experiment that they would earn 1 point for each "correct" response (i.e. correct detection when the morph level was up to 3), that could result, depending of their final score, in a bonus of 5 to 10 euros. Feedback of their performance (i.e. percentage of correct responses) was displayed on the screen after each block. 
1 Every 8 blocks, a progress bar appeared that allowed participants to follow their progress and to

2 estimate how close they were to receiving a bonus. At the end of the experiment, all participants

3 received the same compensation, irrespective of their performance.

\section{$4 \quad$ EEG acquisition and pre-processing}

5 EEG activity was recorded at a sampling rate of $1024 \mathrm{~Hz}$ using a 64-channel ActiveTwo system

6 (BioSemi, The Netherlands, http://www.biosemi.com). An average referencing procedure was used,

7 and impedances were kept under a threshold of $10 \mathrm{k} \Omega$.

Raw EEG data was pre-processed using the Brainstorm toolbox for MATLAB (Tadel, Baillet, Mosher, Pantazis, \& Leahy, 2011). First, raw EEG data was recalculated to average reference, downsampled to $500 \mathrm{~Hz}$ (2ms steps), and band-pass filtered between 1 and $40 \mathrm{~Hz}$ to remove slow (sweating) and high (muscular and line noise) frequency artifacts. Second, EEG data was visually inspected to remove muscle artifacts and to identify noisy electrodes that were interpolated to the average of adjacent electrodes. Third, independent component analysis (ICA) that excluded interpolated electrodes was performed on the continuous data and ICA components capturing eye blink artifacts were rejected. This EEG data was then epoched from $2 \mathrm{~s}$ before to $2 \mathrm{~s}$ after the face stimulus onset (20ms steps) and linearly de-trended. Epochs containing activity exceeding a threshold of $+/-70 \mu \mathrm{V}$ were automatically discarded. Finally, visual inspection was done on the resulting single epochs to manually exclude any remaining trials with artifacts. After trial rejection, remaining trials averaged $975 \pm 46$ trials per subject. The resulting data was resampled to $100 \mathrm{~Hz}$ (10ms steps) from $500 \mathrm{~ms}$ before to $1.5 \mathrm{~s}$ after stimuli onset.

Time frequency decomposition was also performed using a Brainstorm toolbox pipeline. Timefrequency representations (TFRs) of single trials were calculated for each subject. Morlet Wavelet transformation with 3-s time resolution (as calculated by the full width at half maximum; FWHM) was used to calculate spectral power estimates at each point of the time-frequency window ranging from 


\section{Behavioral data analyses}

2

3

4

First, repeated-measures ANOVA was performed on the average reaction times with the decision type (emotion or color) and the strength of perceptual evidence ( 8 from 0 for neutral or grey to 7 for anger or violet) as within-subjects factors (see results and Figure 2a).

Second, we performed model-guided analyses of the behavioral data. The SDT theoretical framework distinguishes between sensitivity to sensory information, and response bias (or criterion) that reflects the observer's tendency to interpret the interlocutor as displaying either of the two options (anger or neutral in emotion decisions, violet or grey in color decisions). Within this framework, we fitted participant behavior using a model of choice hypothesizing that decisions are formed on the basis of a noisy comparison between the displayed emotion or color and a criterion. For emotion and color decisions separately, we fitted the data with the simplest model (model 0) that could account for each subject's decisions using a noisy, 'signal detection'-like psychometric model to which we included a lapse rate, thereby considering that subjects guessed randomly on a certain proportion of trials:

$$
\begin{aligned}
& P(\text { anger })=\left[\omega_{\text {emo }} * x_{\text {emo }}+b_{\text {emo }}\right] *(1-\varepsilon)+0.5 * \varepsilon \\
& P(\text { violet })=\left[\omega_{\text {colr }} * x_{\text {colr }}+b_{\text {colr }}\right] *(1-\varepsilon)+0.5 * \varepsilon
\end{aligned}
$$

where $P($ anger $)$ and $P$ (violet) corresponds to the probability of judging the face as angry or violet respectively, (.) to the cumulative normal function, $\omega_{\text {emo }}$ and $\omega$ colr to the perceptual sensitivity to the displayed emotion or color, $x_{e m o}$ and $x_{\text {colr }}$ to a trial-wise array of evidence values in favor of anger (emotion strength in the stimulus) or of violet (color strength in the stimulus) (from 0 for neutral to +7 for an intense display), $b_{e m o}$ and $b_{\text {colr }}$ to an additive, stimulus-independent bias toward one of the neutral/anger choice or grey/violet choice, and $\varepsilon$ to the proportion of random guesses among choices. Sensitivity $\left(\omega_{\text {emo }}, \omega_{\text {colr }}\right)$ and bias $\left(b_{\text {emo }}, b_{\text {colr }}\right)$ parameters computed with these models were extracted and compared using t-test analyses (see results and Figure $2 \mathrm{~b}$ ).

Finally, we assessed the presence of 'interference' effects between stimulus dimensions, by examining whether the decision-irrelevant stimulus dimension (i.e., emotion evidence in color 
1 decisions, and color evidence in emotion decisions) exerted influence on: 1 . the processing of

2 decision-relevant cues (Figure 2cd), and 2. participants' subsequent choice (Figure 2e). We further

3 measured reaction times as well as sensitivity and bias model parameters (computed using the same

4 equation as above) as a function of the irrelevant stimulus dimension (high vs. low) and performed t-

5 test analyses for emotion and color decisions separately (see results and Figure $2 \mathrm{~cd}$ ).

\section{$6 \quad$ EEG statistical analyses}

\section{$7 \quad$ Neural encoding analysis of broadband single-trial EEG signals}

Trial-to-trial fluctuations in broadband neural signals were regressed against model-based quantities of interest (e.g., the amount of evidence information provided by the stimulus) to estimate their 'neural encoding' in EEG activity. In the present detection tasks, evidence strength corresponds to the intensity of the displayed emotion or color mask. On the basis of recent studies (El Zein et al., 2015b; Patron, Mennella, Messerotti Benvenuti, \& Thayer, 2019; Wyart, Myers, \& Summerfield, 2015; Wyart, Nobre, \& Summerfield, 2012a), instead of computing event-related averages, we performed single-trial regressions of EEG signals against these variables. As the detection criterion (bias parameter) was different between the emotion and color tasks (see Results), we wanted to investigate differences in the neural encoding of perceptual evidence for emotion and color independently of participants' subsequent detection of anger or violet. Thus, a general linear regression model (GLM) was first used where the decision-relevant evidence strength (morph levels from level 0 to 7 , centered) and the detection responses ("seen" 1 or "not seen" -1) were introduced as a trial-per-trial predictor of broadband EEG signals at each time point $(t)$ after stimulus onset (from $200 \mathrm{~ms}$ before to $1 \mathrm{~s}$ after stimulus onset), at each electrode (e), separately for emotion and color decision tasks.

$$
E E G_{t, e}=\beta_{0}+\beta_{1} \cdot \text { strength }+\beta_{2} \cdot \text { detection }
$$

where $\beta_{0}, \beta_{1}$ and $\beta_{2}$ are the estimated parameters from the GLM. Note that, in this approach, variability in neural signals is considered as a tool, rather than a nuisance, to unravel the neural correlates of perceptual decision making. All regression-based analyses of the EEG data were 
1 performed independently, for each participant and the corresponding parameter estimates of the

2 regressions, reported in arbitrary units, were extracted. The time course of the "strength" parameter

3 estimate $\beta_{1}$ describes the neural encoding of either the emotion or color information provided by the

4 presented stimulus, controlled for participants' detection performance. To test for the presence of

5 interference effects between stimulus dimensions on the neural encoding of decision evidence, we

6 performed an additional analysis which consisted in adding, to the above-described model, the

7 decision-irrelevant evidence strength (color morph levels for emotion decisions and emotion morph

8 levels for color decisions, centered) as trial-wise predictor of broadband EEG signals.

9 Second, analyses at the group level were employed to test the significance of evidence strength

effects between emotion and color decisions across participants using a standard cluster-based

permutation test $\left(\beta_{\text {emo }}-\beta_{\text {colr }}\right)$. This consisted in: 1 . running standard parametric tests against zero

at each time point, 2. extracting contiguous spatial, temporal or spatiotemporal clusters of

uncorrected significance, and 3. controlling for the type-1 'false alarm' error rate (family wise

correction, i.e. FWER) arising from multiple comparisons through cluster-level statistics using the

Fieldtrip toolbox for MATLAB (Oostenveld, Fries, Maris, \& Schoffelen, 2011) and the procedure

described in (Maris \& Oostenveld, 2007). We identified clusters using an uncorrected p-value level of

0.005. Cluster-level significance was then calculated using the Monte-Carlo method, using a

corrected p-value level of 0.05 . More precisely, the pairing between the experimental conditions and

EEG signals was shuffled pseudo-randomly 1500 times, and the maximal cluster-level statistics were extracted for each shuffle to compute a "null" distribution of effect size across the whole-time window. The proportion of clusters in the null distribution whose statistics exceeded the one obtained for each significant cluster in the original (non-shuffled) data was computed, resulting in its cluster-corrected $p$-value.

This second-level cluster analysis permitted the detection of two spatiotemporal clusters, where the neural encoding of emotion and color information differed significantly following stimulus presentation (see Results and Figure 3). To plot the time course of effects at the electrodes identified 
1 by the spatiotemporal cluster analysis, we averaged parameter estimates of the regression for

2 identified electrodes separately for the two conditions, and identified temporal clusters using t-test

3 analyses against zero followed by the permutation procedure described above (Maris \& Oostenveld,

4 2007). Importantly, the selection of electrodes (based on the difference between the two conditions)

5 for this second analysis was orthogonal to the significance of each condition against zero.

$6 \quad$ Note that the same analysis pipeline was carried out using the detection parameter estimate

$7 \quad \beta_{2}$ of the GLM to characterize the neural correlates of participants' detection (detected vs. missed

8 contrast), independently of the encoding of stimulus strength. Two resulting detection coding

9 clusters were identified: one over parietal electrodes (CP1, P1, P3, P5, PO7, PO3, O1, Oz, POz, Pz, P2,

P4, P6, PO8, PO4, O2) between 280 and $350 \mathrm{~ms}$ (cluster stat, $t_{\text {clust }}=-477.86, p=0.0013$ ) and one over

frontal electrodes (AF3, F1, F3, F5, F7, FC3, Fpz, Fp2, AF8, AF4, AFz, Fz, F2, F6, F8, FT8) between 280

and $360 \mathrm{~ms}$ (cluster stat, $\mathrm{t}_{\text {clust }}=439.71, \mathrm{p}=0.0013$ ) (suppl. Figure 1 ). T-test against 0 corrected for time

performed for emotion and color separately revealed that these clusters code specifically for color

detection between 220 and $380 \mathrm{~ms}$ over parietal sites ( $t$-test against zero color, $\mathrm{t}_{37}=-6.93, \mathrm{p}_{\mathrm{corr}}=0$ )

and between 230 and $400 \mathrm{~ms}$ over frontal sites ( $t$-test against zero color, $t_{37}=6.66, p_{\text {corr }}=0$ ), but not

for emotion detection (cluster 1 : $t$-test against zero emotion, $t_{37}=-0.77, p_{\text {corr }}=0.34$; cluster 2 : $t$-test

against zero emotion, $t_{37}=-0.71$., $p_{\text {corr }}=0.34$ ) (suppl. Figure 1 ).

\section{Source reconstruction analysis}

Source analysis was performed using the minimum norm estimates option in Brainstorm (Tadel et al., 2011). A source head model consisting of 7002 current dipoles was used to calculate Kernel inversion matrices for each subject based on all the trials of the subject. Dipole orientations were constrained to be normal to the local cortical surface (Size of the inverse operator: [7002 vertices $x$ 64 channels]). The value of the estimated current density was produced using a "depth-weighted" linear L2-minimum norm estimate current density. We then computed sources by multiplying the resulting individual Kernel matrices (7002 vertices * 64 electrodes) by the extracted parameter estimates from our previous GLM using either emotion or color strength as predictors of single trials 
1 EEG signal (64 electrodes * 201 time points) (see regression analysis). We subtracted the resulting

2 cortical sources sustaining the encoding of emotion minus the encoding of color strength and finally

3 displayed this difference on the cortex surface at a threshold of $60 \%$ of the maximum amplitude

4 (spatial smoothing $6 \mathrm{~mm}$ ).

\section{$5 \quad$ Choice prediction using time frequency motor lateralization measures}

$6 \quad$ We predicted that threat displays could impact not only the neural encoding of perceptual

7 evidence, but also the preparation of the upcoming choice in effector selective regions (Conty et al.,

8 2012; El Zein et al., 2015b). We thus measured choice-predictive signals in the neural data by

9 exploiting a commonly used motor lateralization index calculated from spectral power (de Lange, Rahnev, Donner, \& Lau, 2013; Donner, Siegel, Fries, \& Engel, 2009; Wyart, de Gardelle, Scholl, \& Summerfield, 2012b; Wyart et al., 2015) and tested whether and when this index could predict subsequent choices.

We first isolated effector-selective (left vs right hand) neural activity. As mu activity is known to be suppressed at response time in the hemisphere contralateral to the hand used for response (de Lange et al., 2013; Donner et al., 2009), spectral power from 8 to $12 \mathrm{~Hz}$ at response time was calculated, at each electrode and time point for all subjects, for both emotion and color decisions. The resulting response-locked mu activity for the trials where the subjects responded with their right hand was then subtracted from that of the trials where the subjects responded with their left hand. After averaging across all subjects, electrodes where the motor lateralization was maximal at $200 \mathrm{~ms}$ before response time were identified at 'P3,' $\mathrm{CP} 3$ ', 'C3' for the left hemisphere and 'P4,' $\mathrm{CP} 4$,,' $\mathrm{C} 4$ ' for the right hemisphere for both types of decisions (Figure 4a).

We then computed the motor lateralization index based on this effector-selective neural activity, which allowed us to quantify the motor preparation to response 'anger' or 'violet' just after stimulus onset. For emotion decisions, motor lateralization specific to 'anger' responses was obtained by subtracting contralateral from ipsilateral mu spectral activity $(8-12 \mathrm{~Hz})$ relative to the hand assigned to 'anger' response (counterbalanced across subjects), over effector-selective electrodes at each 
1 time point for all subjects. The resulting index is therefore positive around response time for anger

2 responses and negative for neutral responses. To see whether upcoming choices could be predicted

3 by this motor preparation signal at earlier latencies before the response, we applied the previous

4 trial-by-trial GLM analyses approaches by regressing participants' choice (anger or neutral) against

5 the resulting motor lateralization in dex over time, from stimulus onset:

$$
\text { choice }_{(\text {anger or neutral })}=\beta_{0}+\beta_{1} \cdot E E G_{\text {lat } i d x(t, e)}
$$

6 We extracted and plotted the parameter estimates of this regression throughout time (Figure

74 4b). For color decisions, the exact same analysis procedure was applied to 'grey' or 'violet' responses.

8 T-tests against zero, corrected for time by permutation procedure (Maris \& Oostenveld, 2007), were

9 performed on the parameter estimate $\beta_{1}$ to assess when we could predict the subsequent choice

from the motor lateralization index. We further applied a bootstrapping method to test whether

there is a significant shift in the onset of choice predictability between emotion and color decisions,

using the 'jackknifing' procedure described in (Kiesel, Miller, Jolicoeur, \& Brisson, 2008) (see results and Figure $4 \mathrm{~b})$.

\section{Anxiety states measures}

We measured anxiety prior the experimental session, before data collection, using both the Spielberger State-Trait Anxiety Inventory (STAI) (Spielberger, 1983) and the Liebowitz Social Anxiety Scale (LSAS) (Liebowitz, 1987). The STAI is a self-questionnaire providing a measure of clinical vulnerability for anxiety disorders (Grupe \& Nitschke, 2013). Participant state anxiety scores (STAI Y1) ranged from 20 to 51 (mean $=29.6, S D=7.4)$. Trait anxiety scores (STAI Y-2) ranged from 26 to 64 (mean $=43.6, \mathrm{SD}=10.4)$. The LSAS is a short self-report scale (24 items) assessing fear and avoidance in social interactions (11 items) or social performance (13 items). Example items include, respectively, "Talking to people in authority" and "Entering a room when others are already seated." Items are rated on 4-point scales of fear and avoidance, which range from 0 (none and never, anxiety and avoidance ratings, ranging from 55 to 137 (mean $=87.7, S D=17.9$ ). 
1

Running correlations between these anxiety scores revealed a significant relationship between general trait and state anxiety $(r=0.74, p<0.001)$ as well as between general anxiety traits and social anxiety $(r=0.51, p<0.01)$. Social anxiety scores also tend to correlate with general anxiety state $(r=0.29, p=0.06)$. Therefore, to extract a specific score for social anxiety, corrected for general anxiety, we performed a PCA analysis using the 3 measures of anxiety (LSAS, STAI A and STAI B) and used the 'residual LSAS' - after subtracting the first PCA component - as a regressor used to mediansplit participants into two equally-sized groups in EEG analyses. This removal of the first PCA component, which correlated with all 3 measures of anxiety, allowed us to obtain a more selective measure of participants' social anxiety. As indicated above, we thus performed a median split of our participants in two groups according to the corrected scores ("high" and "low" LSAS), and ran the exact same analyses as described previously to assess whether the encoding of stimulus strength in broadband single-trial EEG signals (see Figure 5a) and choice prediction in time frequency motor lateralization measures (see Figure $5 b$ ) are a function of social anxiety states, specifically during emotion decisions (see also suppl. Figures).

\section{Results}

\section{Behavior}

The decision type (emotion or color) impacted participants' reaction times who were faster to make a decision about color (mean $R T_{\text {colr }}=679 \mathrm{~ms} \pm 16 \mathrm{~ms}$ ) as compared to emotion (mean $R T_{e m o}=798 \mathrm{~ms} \pm 18 \mathrm{~ms}$ ) (repeated-measures ANOVA, $\mathrm{F}_{1,37}=95.3, \mathrm{p}<.0001$, 国 ${ }_{2}=0.72$ ). There was also a main effect of the perceptual evidence strength on participants' reaction times (ANOVA, $F_{1,7}=32.3$, $\mathrm{p}<.0001,]_{2}=0.46-$ participants were faster with strong emotion or color evidence), and an interaction between decision type (emotion or color) and the perceptual evidence strength (ANOVA, $F_{1,7}=19.24, p<.0001$, ? $_{2}=0.34$ ) (see Figure $2 a$ ).

To check the efficiency of the titration procedure in equalizing detection sensitivity (decision difficulty) across the two stimulus dimensions (emotion and color), we then fitted participant behavior, separately for emotion and color decisions, using a classical 'signal detection'-like 
1 psychometric model of decision. Two parameters were computed: the sensitivity to sensory cue $\omega$

2 emo/colr and the shift of the decision towards one choice (bias or criterion) $b$ emo/colr (see method).

3 Thanks to the titration procedure, the detection sensitivity parameters were indeed similar across

4 emotion and color decisions $\left(t_{37}=0.46, p=0.64\right)$, confirming that the tasks matched in terms of

5 difficulty (Figure 2). The detection criterion (bias parameter) was however different between the

6 emotion and color tasks despite matched sensitivity $\left(t_{37}=2.64, p=0.012\right)$ (Figure 2$)$. The difference in

7 detection criterion indicated that less evidence was required to answer anger, i.e. there was a

8 stronger shift of the decision towards anger than toward violet, which we controlled for in all brain

9 analyses (see Methods).

10 Furthermore, we tested whether decision-irrelevant stimulus dimension interfered with the processing of decision-relevant cues (Figure $2 \mathrm{~cd}$ ). One could indeed assume that highly salient stimuli such as angry facial expression may have captured attention and transiently interfered with the processing of color information. However, our results showed that the decision-irrelevant stimulus dimension did not impact reaction times during either emotion decisions ( $\mathrm{t}$-test high vs. low color, $t_{37}=0.32, p=0.74$ ) or color decisions (t-test high vs. low emotion, $t_{37}=-0.05, p=0.95$ ) (Figure $2 c$ ). Moreover, neither the perceptual sensitivity model parameters nor the response bias ones were modulated by the irrelevant color information during emotion decisions (t-test high vs low color, $\omega_{\text {emo }}: t_{37}=0.43, p=0.66 ; b_{e m o}: t_{37}=0.21, p=0.83$ ) or by the irrelevant emotion information during color decisions (t-test high vs low emotion, $\omega_{\text {colr }}: t_{37}=-0.65, p=0.51 ; b_{\text {colr }}: t_{37}=-0.68, p=0.49$ ) (Figure 2d). Finally, the strength of decision-irrelevant stimulus dimension did not influence participants' subsequent choice: the proportion of responding 'anger' or 'violet' remained below chance level, irrespective of the strength of the decision-irrelevant 'violet' or 'anger' information, respectively (Figure 2e, see bottom left and top right curves). 
1 Earlier neural encoding of decision evidence in premotor EEG activity during emotion

\section{2 decisions}

3

4

5

6

7

8

9

Using regression of broadband single-trial EEG signals and spatio-temporal cluster correction, we then identified time points and electrodes where the neural 'encoding' of emotion and color information differed following stimulus presentation between emotion and color decisions (see Method). As we looked for evidence of prioritized processing, we focused on early clusters (before $300 \mathrm{~ms}$ ) that disentangle the two types of decisions.

A first negative significant cluster, where parameter estimates diverged between emotion and color decisions, was identified over central electrodes ( $C 1, \mathrm{CP} 1, \mathrm{CPz}, \mathrm{FCz}, \mathrm{Cz}, \mathrm{C} 2, \mathrm{CP} 2)$ between 100 and $210 \mathrm{~ms}$ (emotion vs. color, cluster stat, $\mathrm{t}_{\text {clust }}=-140.79, \mathrm{p}=0.015$ ). The parameter estimates of the regression slope at these electrodes, extracted for emotion and color decisions separately, showed that this early central EEG activity co-varied with emotion strength as early as $50 \mathrm{~ms}$ until $210 \mathrm{~ms}$ (ttest against zero across time, peak: $\mathrm{t}_{37}=-5.21, \mathrm{p}_{\text {corr }}<0.001$ ), but not with color strength ( $\mathrm{t}$-test against zero across time, peak: $\mathrm{t}_{37}=-0.82, \mathrm{p}_{\text {corr }}=0.42$ ) (Figure $3 a$ ).

A second positive significant cluster, where parameter estimates diverge significantly between emotion and color decisions, was revealed over occipito-temporal sites (P7, P9, PO7, O1, Iz, Oz) between 30 and $190 \mathrm{~ms}$ (emotion vs. color, cluster stat, $\mathrm{t}_{\text {clust }}=281.65, \mathrm{p}=0.004$ ). The parameter estimates of the regression slope at these electrodes, extracted for emotion and color decisions separately, showed that this early temporal EEG activity co-varied with emotion strength at a cluster time window between 20 and $200 \mathrm{~ms}$ ( $t$-test against zero emotion across time, peak: $t_{37}=3.36, p_{\text {corr }}=$ 0.002) (Figure 3b). Yet, and contrary to the first negative central cluster, activity in this occipitotemporal cluster was not specific to emotion decisions as it also co-varied with color strength from 40 to $200 \mathrm{~ms}$ (t-test against zero color across time, peak: $\mathrm{t}_{37}=-3.95, \mathrm{p}_{\mathrm{corr}}<0.001$ ) (Figure $3 \mathrm{ab}$ ).

We then assessed potential interference effects between stimulus dimensions on the neural encoding of decision evidence. Neither the activity in the central cluster nor in the temporal one, where the neural encoding of decision-relevant emotion and color information differed (see Results 
1

2

3

4

5

6

7

8

9

and Figure 3), co-varied with the decision-irrelevant evidence strength during emotion decisions (ttest against zero color encoding, cluster $1: t_{37}=0.71, p_{\text {corr }}=0.12$; cluster $2: t_{37}=-0.72$., $p_{\text {corr }}=0.32$ ) (suppl. Figure 2, top line, red curve) or color decisions (t-test against zero emotion encoding, cluster 1:, $\mathrm{t}_{37}=-0.15, \mathrm{p}_{\text {corr }}=0.83$; cluster $2: \mathrm{t}_{37}=0.69 ., \mathrm{p}_{\text {corr }}=0.45$ ) tasks (suppl. Figure 2 , bottom line, yellow curve).

Moreover, emotion decisions were not associated with increased event-related averages compared to color decisions at classical peak latencies for exogenous (P1 measured at PO7, PO3, PO4, PO8, P7, P5, P3, P2, P4, P6, t-test emotion vs. color, average on a statistical significant window 90-140ms: $t_{37}=-2.88, p=0.006$, colr>emo; $\mathrm{N} 1$ measured at PO7, PO3, PO4, PO8, P7, P5, P3, P2, P4, P6, t-test emotion vs. color, average on statistical significant window $160-230 \mathrm{~ms}: t_{37}=0.66, p=0.51$ ) and endogenous ( $\mathrm{P} 3$ measured at $\mathrm{CPz}, \mathrm{Pz}, \mathrm{POZ}$, t-test emotion vs. color, average on statistical significant window 300-670ms: $t_{37}=-0.07, p=0.94$ ) components of attention (Carretie, 2014; Rauss, Pourtois, Vuilleumier, \& Schwartz, 2012).

To identify which brain regions generated the scalp-recorded EEG signals specific to emotion decisions in the identified cluster 1 , we projected the difference between emotion or color parameter estimates from our previous GLM from 100 to $210 \mathrm{~ms}$ on the cortex surface at a threshold of $60 \%$ of the maximum amplitude (spatial smoothing $6 \mathrm{~mm}$ ) (see Methods). The early increase in regression slopes for emotion (compared to color decisions) was sourced in the left hemisphere, in the premotor and motor cortex extending to the inferior frontal gyrus, and in posterior occipital areas (Figure 3c, threshold fixed at $60 \%$ of max amplitude).

\section{Earlier choice-predictive motor preparation in band-limited EEG power during emotion decisions}

Analyses of the neural data had so far indicated that perceptual decision-making on emotion cues is associated with an early enhanced neural encoding of sensory evidence over premotor and sensory cortices as compared to decisions on non-social color cues. To further assess the specificity of the mechanisms engaged during emotion and color decisions, we tested to what extent fluctuations in 
1

2

3

4

5

6

7

8

9

motor-preparatory EEG signals in the mu frequency band $(8-12 \mathrm{~Hz})$ were predictive of participant choice, i.e. more precisely whether and when choices can be decoded from a commonly used motor lateralization index (contralateral-ipsilateral to 'anger' or 'violet' button) (see methods) (de Lange et al., 2013; Donner et al., 2009; Wyart et al., 2012b; Wyart et al., 2015).

Regressing participant choice against this motor lateralization index using GLM, we first showed that the amplitude of the motor-preparatory EEG signals were predictive of participant choice before response time, from $260 \mathrm{~ms}$ after stimulus onset during emotion decisions (t-test against $0, t_{37}=4.29$, $p_{c o r r}<0.001$ ) and from 500ms following stimulus presentation during color decisions (t-test against 0 , $t_{37}=3.87, p_{c o r r}<0.001$ ) (Figure $4 b$ ). To further investigate whether emotional stimuli are prioritized during decision-making, we tested whether the above-described shift in the onset of choice predictability between emotion and color decisions was significant using the 'jackknifing' procedure (Kiesel et al., 2008). This analysis confirmed that the cluster that included participant responses (mean RT= $739 \pm 18 \mathrm{~ms}$ ) differed in its onset between emotion $(260 \mathrm{~ms})$ and color $(500 \mathrm{~ms})$ decisions (latency jackknifed, $\mathrm{t}_{37}=-2.61, \mathrm{p}_{\text {corr }}=0.013$ ) (Figure $4 \mathrm{~b}$ ). This result indicated that, during emotion decisions, the neural competition between the two possible perceptual choices was resolved faster, as early as $260 \mathrm{~ms}$, whereas choice-predictive signals only appeared $200 \mathrm{~ms}$ later during color decisions.

Influence of social anxiety on the early encoding of decision evidence and choicepredictive motor preparation during emotion decisions.

Finally, to further validate our assumption that the neural mechanisms underlying emotion decisions are not only prioritized over non-social decisions, but also specific to this type of decision, we investigated the impact of social anxiety on the above-described specific effects, i.e. the earlier enhanced neural encoding of emotion evidence and earlier choice-predictive activity in premotor regions during emotion decisions. To identify the unique influence of social anxiety on socioemotional information processing, we corrected participants' social anxiety scores for general anxiety using a PCA-based analysis (see Methods), before splitting our participants into two equally- 
1 sized groups based on social anxiety scores. There were no between-group differences in perceptual

2 sensitivity to either emotion (two-sample t-test, $t_{36}=1.01, p=0.32$ ) or color strength (two-sample t-

3 test, $t_{36}=2.01, p=0.06$ ), or in average response times (two sample $t$-test, high LSAS against low LSAS,

$\left.4 \quad t_{36}=-0.91, p=0.37\right)$.

5 First, the early and specific neural sensitivity to emotion evidence in the previously identified

6 premotor cluster 1 (Figure 3a) was significantly modulated by social anxiety (Figure 5a). Only high

7 socially anxious individuals encoded emotion evidence within the premotor cluster window at an

8 early stage from 60 to $220 \mathrm{~ms}$ ( $\mathrm{t}$-test high LSAS against $0, \mathrm{t}_{18}=3.78, \mathrm{p}_{\text {corr }}=0.001$ ) and, crucially, this

9 premotor encoding was stronger in high compared to low socially anxious individuals from $180 \mathrm{~ms}$

until $210 \mathrm{~ms}$ after face onset (two sample t-test high LSAS against low LSAS, peak: $t_{36}=-2.13, p_{\text {corr }}=$

0.041) (Figure 5a, curve). Importantly, no such difference was observed between groups during color

decisions (two sample t-test high LSAS against low LSAS, $\mathrm{t}_{36}=-0.47, \mathrm{p}_{\text {corr }}=0.64$ ) (suppl. Figure 3 ). In

contrast, the early neural encoding of sensory evidence in the occipito-temporal cluster, found in

against low LSAS, $t_{36}=0.42, p_{\text {corr }}=0.67$ ) (suppl. Figure 4 ).

Second, while only a moderate difference between LSAS groups was found in the amplitude of motor preparation EEG signals from $600 \mathrm{~ms}$ (two sample $t$-test, high LSAS against low $L S A S, t_{36}=1.94$, puncorr $=0.06$ ), there was a difference in the onset of choice predictability between high and low socially anxious participants. Fluctuations in the motor-preparatory EEG signals predicted emotion choices earlier, from $420 \mathrm{~ms}$ post-stimuli, in high socially anxious participants compared to low socially anxious participants (choices decoded from $720 \mathrm{~ms}$, latency jackknifed, $\mathrm{t}_{37}=-2.16, \mathrm{p}_{\text {corr }}=0.037$ )

(Figure 5b). Crucially, no significant effect was found between social anxiety groups in the onset of 
1

2

3

4

5

6

7

8

We compared social and non-social decisions using a novel paradigm in which participants performed two different detection tasks (emotion or color) on the same two-dimensional visual stimuli. Our results revealed three specific effects when decisions concern emotions rather than colors. First, a selective early neural encoding of emotion evidence was revealed over the premotor cortex from $100 \mathrm{~ms}$ following stimulus presentation. Second, participant's upcoming emotion choice was decoded earlier from motor-preparatory EEG signals. Third, both the earlier enhanced neural gain of emotion encoding and the earlier choice-predictive activity for emotion decisions were specifically modulated as a function of social anxiety. The present findings, together with the absence of interference effects on behavioral and neural measures, suggest selective and prioritized neural computations of socially-relevant signals rather than biased visual attention toward these sociallyrelevant signals during perceptual decision-making.

Emotion decisions were associated with a specific potentiation over central electrodes, starting at $100 \mathrm{~ms}$ following stimulus presentation. At first sight, a neural encoding of the strength of sociallyrelevant signals in premotor regions may appear surprising. Indeed, although previous decisionmaking experiments that used visual stimuli revealed an encoding of the stimulus sensory properties (i.e. face vs. house) in the occipital and parietal cortices, around $150 \mathrm{~ms}$ post-stimulus (Delis et al., 2016; Tu et al., 2017), most of them failed to find sensory-related activity in the premotor cortex (Boussaoud \& Wise, 1993; Coallier, Michelet, \& Kalaska, 2015; di Pellegrino \& Wise, 1991; Nakayama, Yamagata, Tanji, \& Hoshi, 2008; Wang et al., 2019). Rather, they showed that the premotor plays a key role in the conversion of sensory information into a choice-related signal (Cisek \& Kalaska, 2002; Donner et al., 2009; Wang et al., 2019). The present neural stimulus sensitivity over premotor regions may therefore reflect the integration of a flow of sensory information from occipito-temporal regions with decision signals (upcoming choice) emerging simultaneously in premotor cortices (Cisek \& Kalaska, 2010; Shushruth et al., 2018; Siegel et al., 2015; van Ede et al., 2019). However, the question as to why only socially-relevant signals potentiated early premotor activity remains. 
1

2

3

4

5

6

7

8

9

One likely explanation is related to the behavioral relevance of social signals, especially of emotional expressions. Evolutionary accounts of emotional displays argue that the very function of emotions is to serve communication purposes by conveying critical information about the emitters (Fridlund, 1994; Keltner, 1999), while also facilitating behavioral responses in the observers (Dezecache, Jacob, \& Grèzes, 2015; Dezecache, Mercier, \& Scott-Phillips, 2013). A growing body of literature concurs with evolutionary accounts by highlighting a functional and anatomical link between neural systems that sustain emotional appraisal and those that underlie action preparation (de Gelder, Snyder, Greve, Gerard, \& Hadjikhani, 2004; Grèzes, Valabregue, Gholipour, \& Chevallier, 2014; Pichon, de Gelder, \& Grezes, 2012; Van den Stock, 2011). Moreover, and in agreement with the present results, the influence of emotion stimuli on motor-related areas was observed from 150ms to 300ms post-stimulus onset (Borgomaneri, Gazzola, \& Avenanti, 2014; Conty et al., 2012; EI Zein, Gamond, Conty, \& Grèzes, 2015a; El Zein et al., 2015b; Hortensius, de Gelder, \& Schutter, 2016). We therefore suggest that the early potentiation of premotor activity during emotion decisions is related to the behavioral relevance for the observer of the perceived threat rather than its sensory properties. Such enhanced activations in premotor regions, when confronted with threatsignaling emotions, could promote survival by allowing the selection of adapted behavioral responses in the face of danger (Frijda, 2009; Ohman, Flykt, \& Esteves, 2001; Panksepp, 1998).

Additionally, and similarly to past experiments that observed a build-up of choice-selective activity that depends on the amount of sensory evidence in motor-related regions (Alamia, Zenon, VanRullen, Duque, \& Derosiere, 2019; Chandrasekaran, Peixoto, Newsome, \& Shenoy, 2017; Coallier et al., 2015; de Lange et al., 2013; Derosiere et al., 2018; Donner et al., 2009; Gould, Nobre, Wyart, \& Rushworth, 2012; Thura, Guberman, \& Cisek, 2017; Tosoni et al., 2014; Wyart et al., 2012b), as early as 250 ms after stimulus onset (Acuna \& Pardo-Vazquez, 2011; Chandrasekaran et al., 2017; Peixoto et al., 2018; Thura et al., 2017), we were able to decode participants' choices from the mu/alpha band motor preparation EEG signal. The onset in choice predictive activity was however earlier during emotion decisions ( $260 \mathrm{~ms}$ post stimulus onset) as compared to color decisions (500ms). We 
1 suggest that the behavioral relevance of perceived threat leads to earlier selection between 2 alternatives in premotor cortices.

3 The question arises as to whether the present findings during emotion decisions are dependent 4 upon the behavioral relevance of the social stimuli or upon their salience related to their threatening 5 nature. First, and possibly due to the titration procedure which equalized detection sensitivity across dimensions, highly salient information in the stimulus (angry expressions) did not interfere with processing of color information during color decisions. This indicates that the observed prioritization of socially-relevant signals arose only when these signals were relevant to the task at hand (i.e., during emotion decisions, not color decisions). We therefore propose that our findings can be interpreted as prioritized processing of behaviorally relevant social cues, rather than as a failure of distractor filtering during color decisions. Moreover, we believe that the observed differences between emotion and color decision cannot be explained by increased attention toward emotional cues compared to color ones. Indeed, not only did participants perform the two detection tasks on the same two-dimensional visual stimuli, but classical ERP components known to be strongly modulated by visual attention ('P1' and 'N1') (Carretie, 2014; Rauss et al., 2012) were not enhanced during emotion decisions.

Second, both the earlier potentiation of premotor activity and the earlier choice-predictive activity for emotion decisions were specifically modulated as a function of social anxiety. Classically, high social anxiety levels are associated with excessive fear and concerns of being negatively evaluated by others, leading to anxiety in social situations (APA, 2013). When expressed in moderation, social anxiety can, however, be adaptive by helping individuals not lose sight of what is socially acceptable and what is not (Brosnan, Tone, \& William, 2018; Gilbert, 2001; Leary, 2001). Although socially anxious individuals exhibit attentional biases to threatening and aversive stimuli that are similar to individuals with other anxiety disorders (Bar-Haim, Lamy, Pergamin, BakermansKranenburg, \& van IJzendoorn, 2007), their biases appear to be specific to socially-relevant stimuli 
1

2

3

4

5

6

7

8

9

anxiety was dissociated from general anxiety levels, suggesting that it is the social relevance of the stimuli (rather than another, non-social dimension of the stimuli) which triggered the prioritization of neural computations during emotion decisions.

Nevertheless, the present findings depend on the contrast with a control condition (color) which is neither social nor threatening, and as such cannot differentiate the processing of socio-emotional information from threat-related information in general. Yet, this experimental design allowed participants to perform two different detection tasks (emotion or color) on the same twodimensional visual stimulus, while equalizing detection sensitivity across the two tasks. We propose that the prioritization of emotion decisions is driven the interaction between the social nature of emotions and the behavioral relevance of anger: a social threat which requires a swift behavioral response. Still, further work is needed to complement the present findings, by contrasting social but non-threatening stimuli as well as non-social but threatening stimuli.

Finally, and although we equalized emotion and color decisions in terms of available sensory evidence and decision difficulty, reflected by similar detection sensitivity parameters, a difference in the reaction times and bias parameters was observed between the two types of decision. This difference was reflected by slower reactions times during emotion compared to color decisions and a stronger shift of the decision towards anger than toward violet, which may be due to the fact that failing to correctly identify threat is likely to be more costly than falsely responding in the absence of threat. To address this, we used single-trial regressions of EEG signals against the intensity of the displayed emotion and of the color mask, an experimental approach that measures stimulus sensitivity across tasks and which should dissociate perceptual and response bias. We further controlled all brain analyses on a trial-by-trial basis for the participants' subsequent detection reports (detection of violet in the color task, detection of anger in the emotion task). Yet, recent evidence in mice suggests that a lack of change in sensitivity does not prevent effects on sensory encoding - and that a behavioral bias may betray such an effect (Jin \& Glickfeld, 2019). Therefore, we cannot 
1

2

3

4

5

6

7

completely exclude the possibility that early potentiation of premotor activity during emotion decisions may reflect a better sensory encoding of emotion evidence compared to color evidence.

To conclude, our findings suggest the existence of specialized and prioritized neural computations for processing behaviorally and socially-relevant signals throughout perceptual decision-making. The specific implication of premotor regions during emotion decisions supports the idea that social signals, and particularly emotional displays, are motivationally relevant, not only by providing information about others' affective states and potential behavioral intentions, but more importantly by conveying action demands to the perceiver (Horstmann, 2003).

\section{References}

Acuna, C., \& Pardo-Vazquez, J. L. (2011). Ventral premotor cortex neuronal activity matches perceptual decisions. Eur J Neurosci, 33(12), 2338-2348. doi:10.1111/j.1460-9568.2011.07708.x

Alamia, A., Zenon, A., VanRullen, R., Duque, J., \& Derosiere, G. (2019). Implicit visual cues tune oscillatory motor activity during decision-making. Neuroimage, 186, 424-436. doi:10.1016/j.neuroimage.2018.11.027

APA. (2013). Diagnostic and Statistical Manual of Mental Disorders (Fifth ed.). Arlington, VA American Psychiatric Publishing.

Bar-Haim, Y., Lamy, D., Pergamin, L., Bakermans-Kranenburg, M. J., \& van IJzendoorn, M. H. (2007). Threatrelated attentional bias in anxious and nonanxious individuals: a meta-analytic study. Psychol Bull, 133(1), 1-24. doi:10.1037/0033-2909.133.1.1

Bishop, H. J., Biasini, F. J., \& Stavrinos, D. (2017). Social and Non-social Hazard Response in Drivers with Autism Spectrum Disorder. J Autism Dev Disord, 47(4), 905-917. doi:10.1007/s10803-016-2992-1

Borgomaneri, S., Gazzola, V., \& Avenanti, A. (2014). Temporal dynamics of motor cortex excitability during perception of natural emotional scenes. Soc Cogn Affect Neurosci, 9(10), 1451-1457. doi:10.1093/scan/nst139

Boussaoud, D., \& Wise, S. P. (1993). Primate frontal cortex: effects of stimulus and movement. Exp Brain Res, 95(1), 28-40. doi:10.1007/bf00229651

Brainard, D. H. (1997). The Psychophysics Toolbox. Spat Vis, 10(4), 433-436.

Brosnan, S. F., Tone, E. B., \& William, L. (2018). The Evolution of Social Anxiety. In E. p. T. K. Shackelford \& V. Zeigler-Hill (Eds.) (Ed.), The evolution of psychopathology (pp. 93-116). Cham, Switzerland: Springer International Publishing.

Carretie, L. (2014). Exogenous (automatic) attention to emotional stimuli: a review. Cogn Affect Behav Neurosci, 14(4), 1228-1258. doi:10.3758/s13415-014-0270-2

Chandrasekaran, C., Peixoto, D., Newsome, W. T., \& Shenoy, K. V. (2017). Laminar differences in decisionrelated neural activity in dorsal premotor cortex. Nat Commun, 8(1), 614. doi:10.1038/s41467-01700715-0

Cisek, P., \& Kalaska, J. F. (2002). Simultaneous encoding of multiple potential reach directions in dorsal premotor cortex. J Neurophysiol, 87(2), 1149-1154. doi:10.1152/jn.00443.2001

Cisek, P., \& Kalaska, J. F. (2010). Neural mechanisms for interacting with a world full of action choices. Annu Rev Neurosci, 33, 269-298. doi:10.1146/annurev.neuro.051508.135409 
Coallier, E., Michelet, T., \& Kalaska, J. F. (2015). Dorsal premotor cortex: neural correlates of reach target decisions based on a color-location matching rule and conflicting sensory evidence. J Neurophysiol, 113(10), 3543-3573. doi:10.1152/jn.00166.2014

Conty, L., Dezecache, G., Hugueville, L., \& Grèzes, J. (2012). Early binding of gaze, gesture, and emotion: neural time course and correlates. J Neurosci, 32(13), 4531-4539. doi:10.1523/JNEUROSCl.5636-11.2012

de Gelder, B., Snyder, J., Greve, D., Gerard, G., \& Hadjikhani, N. (2004). Fear fosters flight: a mechanism for fear contagion when perceiving emotion expressed by a whole body. Proc Natl Acad Sci U S A, 101(47), 16701-16706. doi:10.1073/pnas.0407042101

de Lange, F. P., Rahnev, D. A., Donner, T. H., \& Lau, H. (2013). Prestimulus oscillatory activity over motor cortex reflects perceptual expectations. J Neurosci, 33(4), 1400-1410. doi:10.1523/JNEUROSCI.1094-12.2013

Delis, I., Onken, A., Schyns, P. G., Panzeri, S., \& Philiastides, M. G. (2016). Space-by-time decomposition for single-trial decoding of M/EEG activity. Neuroimage, 133, 504-515. doi:10.1016/j.neuroimage.2016.03.043

Derosiere, G., Klein, P. A., Nozaradan, S., Zenon, A., Mouraux, A., \& Duque, J. (2018). Visuomotor Correlates of Conflict Expectation in the Context of Motor Decisions. J Neurosci, 38(44), 9486-9504. doi:10.1523/JNEUROSCI.0623-18.2018

Dezecache, G., Jacob, P., \& Grèzes, J. (2015). Emotional contagion: its scope and limits. Trends Cogn Sci, 19(6), 297-299. doi:10.1016/j.tics.2015.03.011

Dezecache, G., Mercier, H., \& Scott-Phillips, T. (2013). An Evolutionary Approach to Emotional Communication. SSRN Scholarly Paper No. ID 2345829. Rochester, NY: Social Science Research Network. Retrieved from https://papers.ssrn.com/abstract=2345829.

di Pellegrino, G., \& Wise, S. P. (1991). A neurophysiological comparison of three distinct regions of the primate frontal lobe. Brain, 114 (Pt 2), 951-978. doi:10.1093/brain/114.2.951

Donner, T. H., Siegel, M., Fries, P., \& Engel, A. K. (2009). Buildup of choice-predictive activity in human motor cortex during perceptual decision making. Curr Biol, 19(18), 1581-1585. doi:10.1016/j.cub.2009.07.066

El Zein, M., Gamond, L., Conty, L., \& Grèzes, J. (2015a). Selective attention effects on early integration of social signals: same timing, modulated neural sources. Neuroimage, 106, 182-188. doi:10.1016/j.neuroimage.2014.10.063

El Zein, M., Wyart, V., \& Grèzes, J. (2015b). Anxiety dissociates the adaptive functions of sensory and motor response enhancements to social threats. Elife, 4. doi:10.7554/eLife.10274

Fletcher-Watson, S., Findlay, J. M., Leekam, S. R., \& Benson, V. (2008). Rapid detection of person information in a naturalistic scene. Perception, 37(4), 571-583. doi:10.1068/p5705

Fridlund, A. J. (1994). Human Facial Expression: An Evolutionary View. San Diego: Academic Press Inc.

Frijda, N. H. (2009). Emotion Experience and its Varieties. Emotion Review, 1(3), 264-271. doi:10.1177/1754073909103595

Gilbert, P. (2001). Evolution and social anxiety. The role of attraction, social competition, and social hierarchies. Psychiatr Clin North Am, 24(4), 723-751.

Gould, I. C., Nobre, A. C., Wyart, V., \& Rushworth, M. F. (2012). Effects of decision variables and intraparietal stimulation on sensorimotor oscillatory activity in the human brain. J Neurosci, 32(40), 13805-13818. doi:10.1523/JNEUROSCI.2200-12.2012

Grèzes, J., Valabregue, R., Gholipour, B., \& Chevallier, C. (2014). A direct amygdala-motor pathway for emotional displays to influence action: A diffusion tensor imaging study. Hum Brain Mapp, 35(12), 5974-5983. doi:10.1002/hbm.22598

Grupe, D. W., \& Nitschke, J. B. (2013). Uncertainty and anticipation in anxiety: an integrated neurobiological and psychological perspective. Nat Rev Neurosci, 14(7), 488-501. doi:10.1038/nrn3524

Hanks, T. D., \& Summerfield, C. (2017). Perceptual Decision Making in Rodents, Monkeys, and Humans. Neuron, 93(1), 15-31. doi:10.1016/j.neuron.2016.12.003

Heekeren, H. R., Marrett, S., Bandettini, P. A., \& Ungerleider, L. G. (2004). A general mechanism for perceptual decision-making in the human brain. Nature, 431(7010), 859-862. doi:10.1038/nature02966

Horstmann, G. (2003). What do facial expressions convey: feeling states, behavioral intentions, or action requests? Emotion, 3(2), 150-166.

Hortensius, R., de Gelder, B., \& Schutter, D. J. (2016). When anger dominates the mind: Increased motor corticospinal excitability in the face of threat. Psychophysiology, 53(9), 1307-1316. doi:10.1111/psyp.12685

Jin, M., \& Glickfeld, L. L. (2019). Contribution of Sensory Encoding to Measured Bias. J Neurosci, 39(26), 51155127. doi:10.1523/JNEUROSCl.0076-19.2019 
Keltner, D., \& Haidt, J. (1999). Social Functions of Emotions at Four Levels of Analysis. Cognition and Emotion, 13(5), 505-521. doi:10.1080/026999399379168

Kiesel, A., Miller, J., Jolicoeur, P., \& Brisson, B. (2008). Measurement of ERP latency differences: a comparison of single-participant and jackknife-based scoring methods. Psychophysiology, 45(2), 250-274. doi:10.1111/j.1469-8986.2007.00618.x

Kraus, J., Frick, A., Fischer, H., Howner, K., Fredrikson, M., \& Furmark, T. (2018). Amygdala reactivity and connectivity during social and non-social aversive stimulation in social anxiety disorder. Psychiatry Res Neuroimaging, 280, 56-61. doi:10.1016/j.pscychresns.2018.08.012

Langner, O., Dotsch, R., Bijlstra, G., Wigboldus, D., Hawk, S. T., \& van Knippenberg, A. (2010). Presentation and validation of the Radboud Faces Database. Cognition and Emotion, 24(8). doi:10.1080/02699930903485076

Leary, M. R. (2001). Social anxiety as an early warning system: A refinement and extension of the selfpresentation theory of social anxiety. In P. M. D. S. G. Hofmann, S. G. Hofmann, \& P. M. DiBartolo (Eds.) (Ed.), From social anxiety to social phobia: Multiple perspectives (pp. 321-334). Needham Heights, MA: Allyn \& Bacon.

Liebowitz, M. (1987). Social phobia. . Modern Problems in Pharmacopsychiatry, 22, 141-173.

Maris, E., \& Oostenveld, R. (2007). Nonparametric statistical testing of EEG-and MEG-data. J Neurosci Methods, 164(1), 177-190. doi:10.1016/j.jneumeth.2007.03.024

Nakayama, Y., Yamagata, T., Tanji, J., \& Hoshi, E. (2008). Transformation of a virtual action plan into a motor plan in the premotor cortex. J Neurosci, 28(41), 10287-10297. doi:10.1523/JNEUROSCI.2372-08.2008

Ohman, A., Flykt, A., \& Esteves, F. (2001). Emotion drives attention: detecting the snake in the grass. J Exp Psychol Gen, 130(3), 466-478.

Oostenveld, R., Fries, P., Maris, E., \& Schoffelen, J. M. (2011). FieldTrip: Open source software for advanced analysis of MEG, EEG, and invasive electrophysiological data. Comput Intell Neurosci, 2011, 156869. doi:10.1155/2011/156869

Panksepp, J. (1998). Affective neuroscience: The foundations of human and animal emotions. Oxford UK: Oxford University Press.

Patron, E., Mennella, R., Messerotti Benvenuti, S., \& Thayer, J. F. (2019). The frontal cortex is a heart-brake: Reduction in delta oscillations is associated with heart rate deceleration. Neuroimage, 188, 403-410. doi:10.1016/j.neuroimage.2018.12.035

Peixoto, D., Kiani, R., Chandrasekaran, C., Ryu, S., Shenoy, K. V., \& Newsome, W. T. (2018). Population dynamics of choice representation in 2 dorsal premotor and primary motor cortex. bioRxiv. doi:10.1101/283960

Pelli, D. G. (1997). The VideoToolbox software for visual psychophysics: transforming numbers into movies. Spat Vis, 10(4), 437-442.

Pessoa, L., \& Padmala, S. (2005). Quantitative prediction of perceptual decisions during near-threshold fear detection. Proc Natl Acad Sci U S A, 102(15), 5612-5617. doi:10.1073/pnas.0500566102

Pessoa, L., \& Padmala, S. (2007). Decoding near-threshold perception of fear from distributed single-trial brain activation. Cereb Cortex, 17(3), 691-701. doi:10.1093/cercor/bhk020

Philiastides, M. G., Ratcliff, R., \& Sajda, P. (2006). Neural representation of task difficulty and decision making during perceptual categorization: a timing diagram. J Neurosci, 26(35), 8965-8975. doi:10.1523/JNEUROSCI.1655-06.2006

Philiastides, M. G., \& Sajda, P. (2006). Temporal characterization of the neural correlates of perceptual decision making in the human brain. Cereb Cortex, 16(4), 509-518. doi:10.1093/cercor/bhi130

Philiastides, M. G., \& Sajda, P. (2007). EEG-informed fMRI reveals spatiotemporal characteristics of perceptual decision making. J Neurosci, 27(48), 13082-13091. doi:10.1523/JNEUROSCI.3540-07.2007

Pichon, S., de Gelder, B., \& Grezes, J. (2012). Threat prompts defensive brain responses independently of attentional control. Cereb Cortex, 22(2), 274-285. doi:10.1093/cercor/bhr060

Rauss, K., Pourtois, G., Vuilleumier, P., \& Schwartz, S. (2012). Effects of attentional load on early visual processing depend on stimulus timing. Hum Brain Mapp, 33(1), 63-74. doi:10.1002/hbm.21193

Ro, T., Russell, C., \& Lavie, N. (2001). Changing faces: a detection advantage in the flicker paradigm. Psychol Sci, 12(1), 94-99. doi:10.1111/1467-9280.00317

Sander, D., Grandjean, D., Kaiser, S., Wehrle, T., \& Scherer, K. R. (2007). Interaction effects of perceived gaze direction and dynamic facial expression: Evidence for appraisal theories of emotion. European Journal of Cognitive Psychology, 19(3), 470-480. doi:10.1080/09541440600757426

Shultz, S., Klin, A., \& Jones, W. (2018). Neonatal Transitions in Social Behavior and Their Implications for Autism. Trends Cogn Sci, 22(5), 452-469. doi:10.1016/j.tics.2018.02.012 


\author{
Shushruth, S., Mazurek, M., \& Shadlen, M. N. (2018). Comparison of Decision-Related Signals in Sensory and \\ Motor Preparatory Responses of Neurons in Area LIP. J Neurosci, 38(28), 6350-6365. \\ doi:10.1523/JNEUROSCI.0668-18.2018 \\ Siegel, M., Buschman, T. J., \& Miller, E. K. (2015). Cortical information flow during flexible sensorimotor \\ decisions. Science, 348(6241), 1352-1355. doi:10.1126/science.aab0551 \\ Spielberger, C. D., Gorsuch, R. L., Lushene, R., Vagg, P. R., \& Jacobs, G. A. . (1983). Manual for the State-Trait \\ Anxiety Inventory. Palo Alto, CA: Consulting Psychologists Press. \\ Sun, S., Yu, R., \& Wang, S. (2017). A Neural Signature Encoding Decisions under Perceptual Ambiguity. eNeuro, \\ 4(6). doi:10.1523/ENEURO.0235-17.2017 \\ Tadel, F., Baillet, S., Mosher, J. C., Pantazis, D., \& Leahy, R. M. (2011). Brainstorm: a user-friendly application for \\ MEG/EEG analysis. Comput Intell Neurosci, 2011, 879716. doi:10.1155/2011/879716 \\ Thielscher, A., \& Pessoa, L. (2007). Neural correlates of perceptual choice and decision making during fear- \\ disgust discrimination. J Neurosci, 27(11), 2908-2917. doi:10.1523/JNEUROSCl.3024-06.2007 \\ Thura, D., Guberman, G., \& Cisek, P. (2017). Trial-to-trial adjustments of speed-accuracy trade-offs in premotor \\ and primary motor cortex. J Neurophysiol, 117(2), 665-683. doi:10.1152/jn.00726.2016 \\ Tosoni, A., Corbetta, M., Calluso, C., Committeri, G., Pezzulo, G., Romani, G. L., \& Galati, G. (2014). Decision and \\ action planning signals in human posterior parietal cortex during delayed perceptual choices. Eur J \\ Neurosci, 39(8), 1370-1383. doi:10.1111/ejn.12511 \\ Tu, T., Schneck, N., Muraskin, J., \& Sajda, P. (2017). Network Configurations in the Human Brain Reflect Choice \\ Bias during Rapid Face Processing. J Neurosci, 37(50), 12226-12237. doi:10.1523/JNEUROSCl.1677- \\ 17.2017 \\ Van den Stock, J., Tamietto, M., Sorger, B., Pichon, S., Grézes, J., \& de Gelder, B. (2011). Corticosubcortical \\ visual, somatosensory, and motor activations for perceiving dynamic whole-body emotional \\ expressions with and without striate cortex (V1). Proceedings of the NationalAcademy of Sciences, \\ 108(39), 16188-16193. \\ van Ede, F., Chekroud, S. R., Stokes, M. G., \& Nobre, A. C. (2019). Concurrent visual and motor selection during \\ visual working memory guided action. Nat Neurosci, 22(3), 477-483. doi:10.1038/s41593-018-0335-6 \\ Vuilleumier, P. (2005). How brains beware: neural mechanisms of emotional attention. Trends Cogn Sci, 9(12), \\ 585-594. doi:10.1016/j.tics.2005.10.011 \\ Wang, M., Montanede, C., Chandrasekaran, C., Peixoto, D., Shenoy, K. V., \& Kalaska, J. F. (2019). Macaque \\ dorsal premotor cortex exhibits decision-related activity only when specific stimulus-response \\ associations are known. Nat Commun, 10(1), 1793. doi:10.1038/s41467-019-09460-y \\ Wyart, V., de Gardelle, V., Scholl, J., \& Summerfield, C. (2012b). Rhythmic fluctuations in evidence \\ accumulation during decision making in the human brain. Neuron, 76(4), 847-858. \\ doi:10.1016/j.neuron.2012.09.015 \\ Wyart, V., Myers, N. E., \& Summerfield, C. (2015). Neural mechanisms of human perceptual choice under \\ focused and divided attention. J Neurosci, 35(8), 3485-3498. doi:10.1523/JNEUROSCI.3276-14.2015 \\ Wyart, V., Nobre, A. C., \& Summerfield, C. (2012a). Dissociable prior influences of signal probability and \\ relevance on visual contrast sensitivity. Proc Natl Acad Sci U S A, 109(9), 3593-3598. \\ doi:10.1073/pnas.1120118109
}




\section{Figure Captions}

2 Figure 1. Stimuli and experimental procedures. The top panel represents examples of morphed anger expressions and color masks used to orthogonally manipulate the strength of perceptual evidence in the stimuli. Note that only four of the seven levels of morphs created from neutral to anger and from grey to violet are presented. The bottom panel illustrates the time course of one trial during perceptual detection tasks. After a fixation period, a morphed face, on top of which is superposed a morphed color mask, was displayed for $250 \mathrm{~ms}$. After face offset, and depending on the block, participants reported the presence or absence of either emotion (anger) or color (violet) in the stimuli, while ignoring the other task-irrelevant dimension.

Figure 2. Behavioral results. (a) Mean reaction times as function of decision type (emotion in blue and color in grey) (left bar plot) and according to decision-relevant evidence strength (right curve). In general, participants were faster with strong emotion or color evidence, and they were the slowest at different levels of perceptual ambiguity in the two tasks (around 0.6 for emotion, and 0.7 for color). (b) Psychometric function representing the proportion $\boldsymbol{P}$ of 'anger' (in blue) or 'violet' (in grey) responses as a function of the perceptual evidence $\boldsymbol{x}$ for anger or violet (proportion morph, $0=$ neutral or grey, $7=100 \%$ anger or violet). Dots and attached error bars indicate the experimental data (mean +/- sem). Lines and shaded error bars indicate the prediction of the fitting model. The bar plots correspond to the bias $\boldsymbol{b}$ (shift from $50 \%$ detection) (right) and the sensitivity $\boldsymbol{\omega}$ (i.e. the slope) (left) parameter estimates of the psychometric curve extracted for each decision type. (c) Reaction times to decision-relevant stimulus dimension (i.e. emotion in emotion decisions and color in color decisions) according to the strength of the decision-irrelevant dimension (high/low color in emotion decisions and high/low emotion in color decisions). Blue dots represent responses to emotion cues when color dimension is high (dark blue) or low (light blue). Red dots represent responses to color cues when emotion dimension is high while yellow dots corresponds to color responses when emotion dimension is low. (d) Psychometric function representing the proportion $\boldsymbol{P}$ of 'anger' or 'violet' responses as a function of the relevant perceptual evidence $\boldsymbol{x}$ for anger or violet, splitted 
1 according to the strength of the irrelevant dimension (high vs. low). Bar plots represent the

2 sensitivity and bias parameters averaged across morph levels. Same convention as in (b) and color

3 codes as in (c). (e) Psychometric function for each dimension (emotion/color) and decision type

4 (emotion/color). Contrary to the relevant stimulus dimension, the strength of the irrelevant

5 dimension did not impact subsequent participants' detection. Same convention as in (b).

6 Figure 3. Neural encoding of decision evidence in (a) central and (b) temporal EEG activity. (a) Scalp

7 topography of a negative cluster over central electrodes (100-210ms), where parameter estimates

8 diverge significantly between emotion and color decisions, and expressed as mean parameter

9 estimates in arbitrary units (a.u.). Dots indicate the electrodes of interest where parameter estimates

diverge significantly between emotion and color decisions. Below the scalp topography, encoding

time course for emotion (blue) and color (grey) decisions separately, at cluster electrodes of interest.

Shaded error bars indicate s.e.m. Shaded light grey area indicates the cluster time window (100-

$210 \mathrm{~ms})$. Blue and grey lines indicate significance against 0 for emotion and color parameter estimates respectively, at p-value 0.05 corrected for time. On the right side of the scalp topography,

the bar plots represent the parameter estimates, for emotion and color decisions separately, averaged across the cluster electrodes of interest and time window. $* * * p<.001$ (b) Same conventions as (a) for a positive cluster over temporal electrodes (30-190ms). (c) Estimated cortical sources of the negative cluster over central electrodes (100-210ms) (see a), where parameter estimates diverge significantly between emotion and color decisions. The estimated sources are mainly located in the left hemisphere, in premotor and motor cortex extending to inferior frontal gyrus, and in posterior occipital areas.

Figure 4. Choice-predictive motor preparation in band-limited EEG power. (a) Lateralized effectorselective cortical activity (left- versus right- hand button press) during the 200ms before choice (response locked) in the mu frequency range $(8-12 \mathrm{~Hz})$. Dots indicate the selected electrodes where the effect was maximal. (b) Time course of choice-predictive activity (stimulus-locked) in the motor 
1 motor-preparatory EEG signals were predictive of participant choices from $260 \mathrm{~ms}$ after stimulus

2 onset during emotion decisions, and only from $500 \mathrm{~ms}$ following stimulus presentation during color

3 decisions (jackknifed). The blue and grey lines indicate significance against 0 for emotion and color

4 task respectively at $p$-value corrected for time. (c) Extracted parameter estimates averaged across

5 the motor lateralization electrodes over the $260-500 \mathrm{~ms}$ window where the shift in the onset of

6 choice predictability between emotion and color decisions was significant, *** $p<.001$.

7 Figure 5. Anxiety-dependent effects during emotion decisions. (a) Neural encoding of decision evidence. Scalp topography of the negative cluster over central electrodes (100-210ms) where parameter estimates were found to diverge significantly between emotion and color decisions (Figure 3a). Below the scalp topography, encoding time course during emotion decisions for High and Low social anxious individuals. Shaded light grey area indicates the cluster time window (100-210ms). The dark grey line, on top of the time course figure, indicates a significant group effect $(2$ sample $t-$ test) at the cluster level corrected for time between 180 and $210 \mathrm{~ms}$. The dark and light blue lines, along the $\mathrm{x}$ axe, indicate significance against 0 for High and Low social anxious groups respectively, at p-value 0.05 corrected for time. On the right side of the scalp topography, the bar plots represent the parameter estimates, for High and Low social anxious groups separately, averaged across the cluster electrodes of interest and time window. ${ }^{* * *} \mathrm{p}<.001$. (b) Choice-predictive motor preparation in band-limited EEG power. The motor preparation signals predict emotion choice earlier, from $420 \mathrm{~ms}$ post-stimuli, in High compared to Low social anxious groups (choices decoded from $720 \mathrm{~ms}$ ), *: $p<0.05$ (jackknifed). The bar plots represent the extracted parameter estimates averaged across the motor preparation electrodes over the $420-720 \mathrm{~ms}$ window, where the shift in the onset of choice predictability between High and Low social anxious groups was significant during emotion decisions. 


\section{Supplementary figures}

3 Suppl. figure 1. Neural correlates of participants' detection (detected vs. missed contrast),

4

5

6 independently of the encoding of stimulus strength. (a) Scalp topography of a negative significant cluster where the detection parameter estimates diverge between emotion and color decisions (280350ms over parietal sites), and expressed as mean parameter estimates in arbitrary units (a.u.). Below the scalp topography, encoding time course for emotion (blue) and color (grey) decisions separately, at cluster electrodes of interest. Shaded error bars indicate s.e.m. Shaded light grey area indicates the cluster time window $(280-350 \mathrm{~ms})$. Blue and grey lines indicate significance against 0 for emotion and color parameter estimates respectively, at $p$-value 0.05 corrected for time. On the right side of the scalp topography, the bar plots represent the parameter estimates, for emotion and color decisions separately, averaged across the cluster electrodes of interest and time window. ${ }^{* * *}$ $\mathrm{p}<.001$. (b) Same conventions as (a) for a positive significant cluster between $280-360 \mathrm{~ms}$ over frontal sites. Note that these two clusters are specific for color detection.

Suppl. figure 2. Neural encoding of decision-irrelevant evidence in central and temporal EEG activity. Curves represent the encoding time course of relevant and irrelevant stimulus evidence for emotion (top line) and color (bottom line) decisions separately, at central (cluster 1) and temporal (cluster 2) electrodes identified in Figure 3. Blue curve represents the neural encoding of emotion cues during emotion decisions while grey curve represents the neural encoding of color cues during color decisions (same as Figure 3). Crucially, red curve indicates that the decision-irrelevant color cues were not encoded during emotion decisions in the 2 clusters (i.e. did not differ from 0 ). Similarly, in color decisions, the decision-irrelevant emotion cues were not encoded in central and temporal EEG activity (yellow curve). Same conventions as for Figure 3.

Suppl. figure 3. Anxiety-independent neural encoding of decision evidence in EEG motor activity during color decisions. Scalp topography of the negative cluster over central electrodes (100-210ms) 
1 where parameter estimates were found to diverge significantly between emotion and color decisions

2 (see Figure 3a). Same conventions as in Figure $5 a$.

3 Suppl. figure 4. Anxiety-independent neural encoding of decision evidence in temporal EEG activity

4 during emotion decisions. Scalp topography of the positive cluster over temporal electrodes (30-

5 190ms) where parameter estimates were found to diverge significantly between emotion and color

6 decisions (see Figure 3a). Conventions are the same as in Figure 5a.

7 Suppl. figure 5. Anxiety-independent choice-predictive motor preparation in band-limited EEG

8 power $(8-12 \mathrm{~Hz}$ ) during color decisions (see also fig.5b). Conventions are the same as in Figure $5 \mathrm{~b}$.

9 


\section{Emotion Decision}

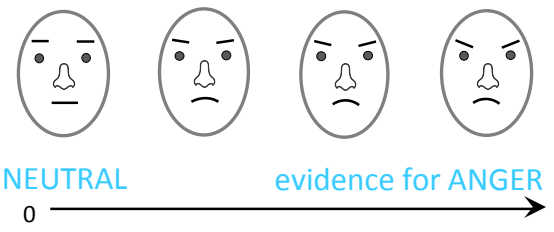

morphed anger expression (7 levels)

\section{Color Decision}

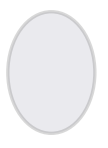

GREY

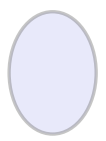

evidence for VIOLET
0

morphed color background (7 levels)
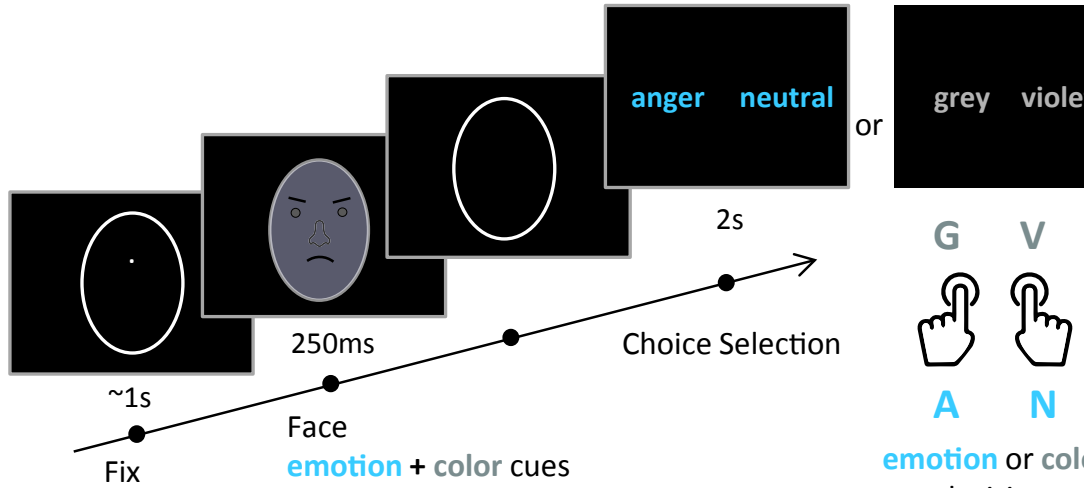

emotion or color decisions 
a.
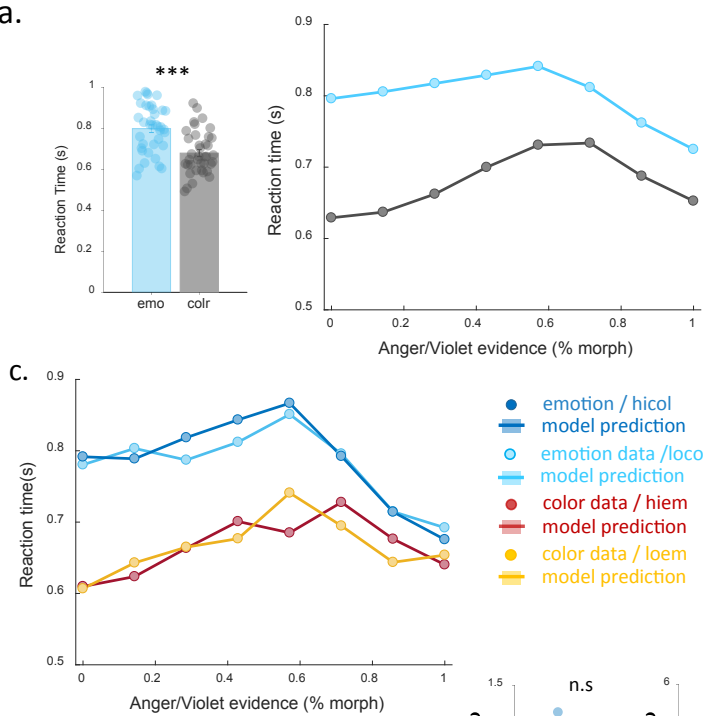

d.

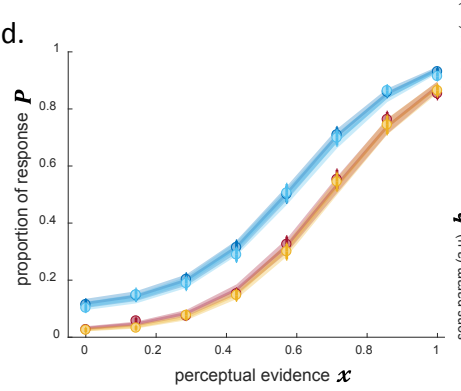

- emotion/hicol

- model prediction

- emotion data/loco

- model prediction

- color data / hiem

- model prediction

- color data / loem

- model prediction

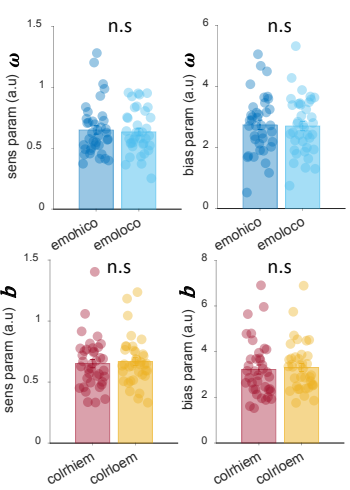

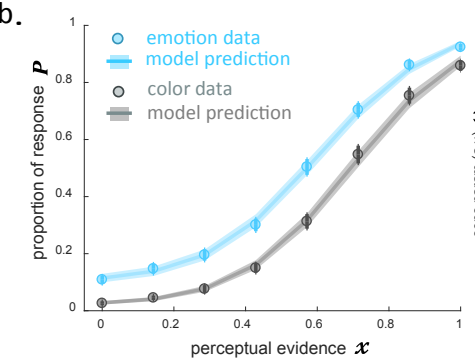
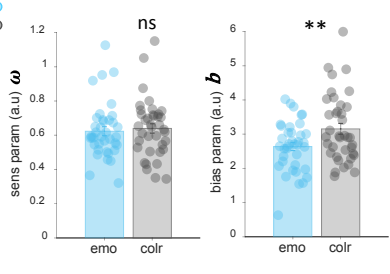

e.

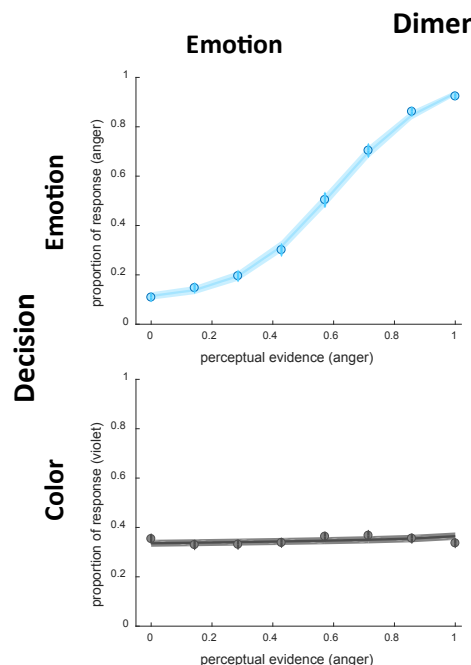

sion
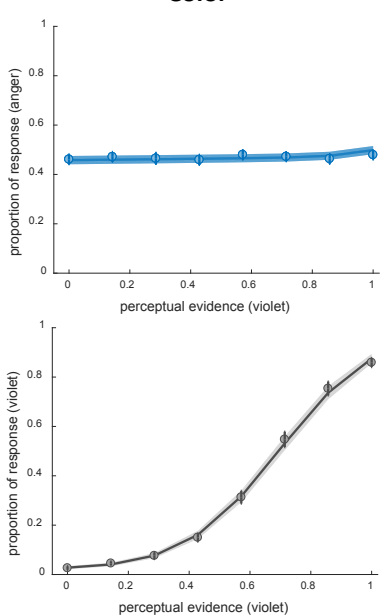


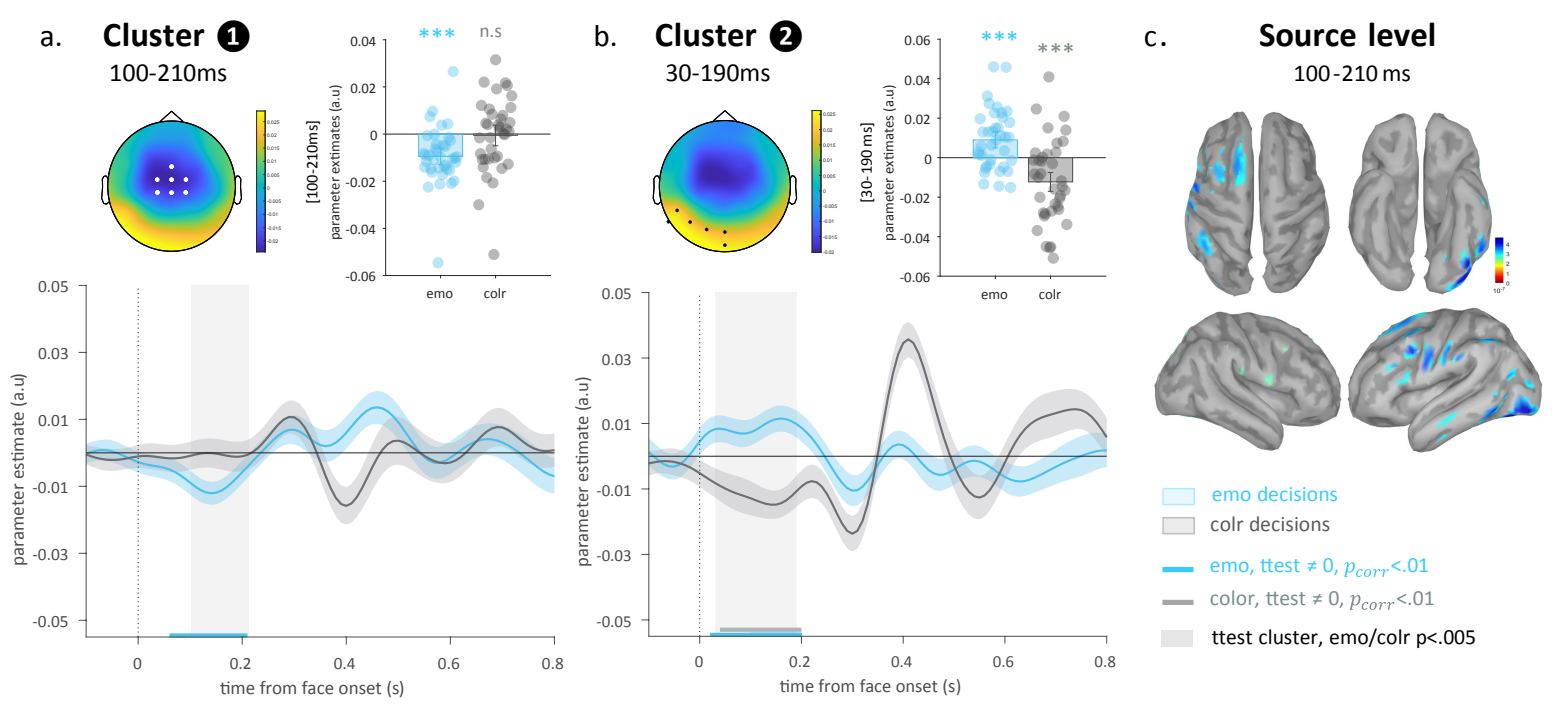




\section{a. Effector-Selective Activity}

Left -Right hand $(8-12 \mathrm{~Hz})$
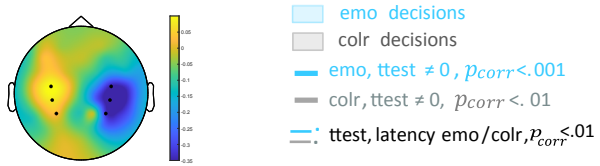

b. Motor lateralization index

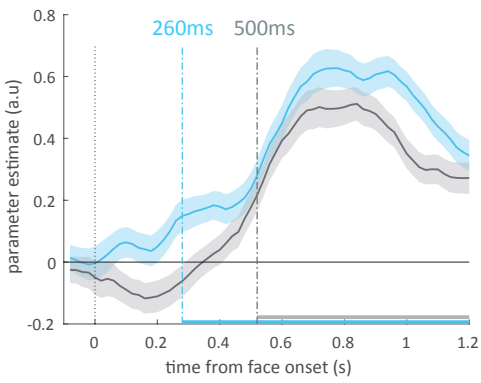

C.

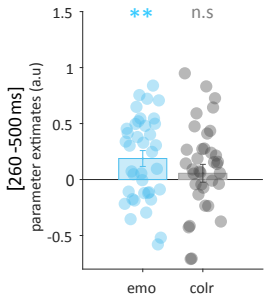




\section{a. Stimulus Strength}

\section{Cluster motor 1}
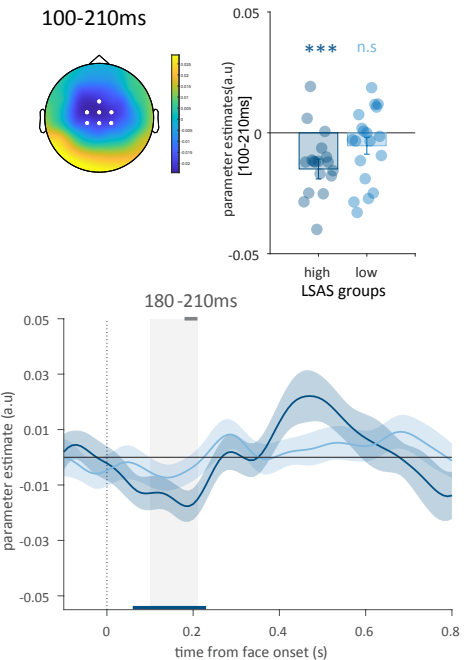

$\square$ High LSAS LOW LSAS

- IoLSAS, ttest $\neq 0, p_{\text {corr }}<.05$

- hilSAS, ttest $\neq 0, p_{\text {corr }}<.05$

\section{b. Choice Prediction}

EffectorSelective

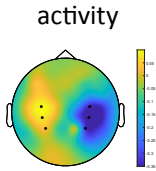

left-right hand
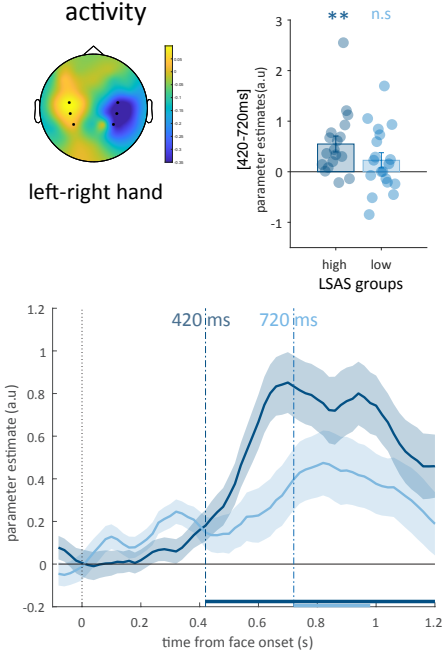

- 2 sample ttest, ampl. hi/lo, $p_{\text {corr }}<0.05$ ttest cluster, emo/colr $\mathrm{p}<.005$

=: ttest, latency hi/lo, $p_{\text {corr }}<.05$ 